\title{
Axially symmetric spacetimes: numerical and analytical perspectives
}

\author{
Sergio Dain \\ Facultad de Matemática, Astronomía y Física, \\ Universidad Nacional de Córdoba, \\ Ciudad Universitaria, (5000) Córdoba, \\ Argentina
}

July 24, 2018

\begin{abstract}
Some new aspects of axially symmetric spacetimes are discussed. These results open the door for future interplay between analytical and numerical studies. The new developments are based on the role of the total mass in axial symmetry. Finally, a list of relevant open problems is presented. These problems can be hopefully solved with an interaction between numerical and analytical insights.
\end{abstract}

\section{Introduction}

In any physical theory, the presence of a symmetry reduces the degrees of freedom of the equations and hence it simplifies considerable the analysis. To study an isolated systems, the simplest models are the spherically symmetric ones. However, it is well known that for vacuum Einstein equations, due to Birkhoff's theorem, spherical symmetric spacetimes has no dynamics. The next possible model with symmetries in vacuum are axially symmetric spacetimes. It has been proved in [4] that no additional symmetry can be imposed to the spacetime if we want to keep the gravitational radiation and a complete null infinity. This result single out axially symmetric spacetimes as the only models for vacuum isolated, dynamical, system with symmetries. From this point of view, axially symmetric gravitational waves are the simplest possible waves emitted by isolated sources. 
There exists many relevant physical models one can study in axial symmetry. In particular, for vacuum and in the strong field regime, we list the following

- Head-on collisions of two black holes.

- Rotating, non-stationary, black holes.

- Formation of black holes: weak cosmic censorship.

- Critical collapse of gravitational waves.

What are the difficulties of axial symmetry? To take advantage of the symmetry an adapted coordinate should be used. However, the reduced equations are formally singular at the axis. This singular behavior introduce a major difficulty in the analysis. In fact, it can be argued that this singular behavior near the axis is so complicated that the axially symmetric case is as hard as the full general case.

What are the advantages of axial symmetry? The first obvious advantage for numerical computations is that axially symmetric spacetimes are less computationally expensive: only two effective spatial dimensions. Also, the number of equations and variables is reduced. The second advantage, which is also well known, is the conservation of angular momentum. The angular momentum is a quasilocal conserved quantity in axial symmetry (Komar integral of the Killing vector). That is, axially symmetric gravitational waves do not carry angular momentum. In particular no Penrose process and no superradiant scattering can occur for axially symmetric vacuum spacetimes. This represents an important simplification in the dynamics. The third advantage is the mass integral formula. The total mass can be written as a positive definite volume integral, as we will see. This is the main new ingredient that we would like to discuss in the following.

The purpose of this review is to summarize some new results for axially symmetric spacetimes with emphasis in the interplay between numerical and analytical studies. The plan of the article is the following. In section 2 we review the axially symmetric equations. The purpose of this section is to present the relevant maximal-isothermal gauge and the corresponding mass formula, which is valid only in this gauge. This formula represents the main new motivation to study axial symmetry. In section 3 we discuss recent results, both numerical and analytical which are based on the maximal-isothermal gauge and the mass formula. Finally, in section 4 we present relevant open problems. 


\section{Axial Symmetry}

Consider a vacuum solution of Einstein's equations, i.e., a four dimensional manifold $M$ with metric $g_{\mu \nu}$ (with signature $(-+++)$ ) such that the corresponding Ricci tensor vanishes

$$
{ }^{(4)} \mathcal{R}_{\mu \nu}=0
$$

Suppose, in addition, that the metric $g_{\mu \nu}$ admits a Killing field $\eta^{\mu}$, that is $\eta^{\mu}$ satisfies the equation

$$
\hat{\nabla}_{(\mu} \eta_{\nu)}=0
$$

where $\hat{\nabla}_{\mu}$ is the connection with respect to $g_{\mu \nu}$. Greek indices $\mu, \nu, \cdots$ denote four dimensional indices.

We define the square of the norm and the twist of $\eta^{\mu}$, respectively, by

$$
\eta=\eta^{\mu} \eta^{\nu} g_{\mu \nu}, \quad \omega_{\mu}=\epsilon_{\mu \nu \lambda \gamma} \eta^{\nu} \hat{\nabla}^{\lambda} \eta^{\gamma} .
$$

Using the field Eq. (1) it is possible to prove that

$$
\hat{\nabla}_{[\mu} \omega_{\nu]}=0
$$

and hence $\omega_{\mu}$ is locally the gradient of a scalar field $\omega$

$$
\omega_{\mu}=\hat{\nabla}_{\mu} \omega .
$$

Let $\mathcal{N}$ denote the collection of all trajectories of $\eta^{\mu}$, and assume that it is a differential 3-manifold. We define the metric $h_{\mu \nu}$ on $\mathcal{N}$ by

$$
\eta g_{\mu \nu}=h_{\mu \nu}+\eta_{\mu} \eta_{\nu} .
$$

The vacuum field equations (1) can be written in the following form on $\mathcal{N}$

$$
\begin{aligned}
\square \eta & =\frac{1}{\eta}\left(\nabla^{a} \eta \nabla_{a} \eta-\nabla^{a} \omega \nabla_{a} \omega\right), \\
\square \omega & =\frac{2}{\eta} \nabla^{a} \omega \nabla_{a} \eta, \\
{ }^{(3)} R_{a b} & =\frac{1}{2 \eta^{2}}\left(\nabla_{a} \eta \nabla_{b} \eta+\nabla_{a} \omega \nabla_{b} \omega\right),
\end{aligned}
$$

where $\nabla_{a}$ and ${ }^{(3)} R_{a b}$ are the connexion and the Ricci tensor of $h_{a b}$, we have defined $\square=\nabla_{a} \nabla^{a}$ and Latin indices $a, b \ldots$ denote three dimensional indices on $\mathcal{N}$. 
Up to this point, the only assumption we have made is that the spacetime admits a Killing vector field $\eta^{\mu}$ and that $\eta^{\mu}$ is not null, otherwise the metric $h_{a b}$ is not defined. If the Killing field is timelike $(\eta<0)$ then the metric $h_{a b}$ is Riemannian and the equations (7) -(9) are the stationary Einstein vacuum equations. On the other hand, when the Killing vector is spacelike $(\eta>0)$, the metric $h_{a b}$ is a is a 3-dimensional Lorenzian metric (we chose the signature $(-++))$. In axial symmetry, the Killing vector $\eta^{\mu}$ is spacelike and its norm vanishes at the axis of symmetry. Hence, the equations are formally singular at the axis. This singular behavior at the axis represents the main difficulty to handle these equations.

In the Lorenzian case, Eq. (9) has the form of Einstein equations in three dimensions coupled with effective matter sources produced by $\eta$ and $\omega$. The effective matter Eqs. (7)-(8) imply that the energy-momentum tensor defined in terms of $\eta$ and $\omega$ by

$$
T_{a b}=\frac{1}{2 \eta^{2}}\left(\nabla_{a} \eta \nabla_{b} \eta+\nabla_{a} \omega \nabla_{b} \omega\right)-\frac{1}{4 \eta^{2}} h_{a b}\left(\nabla_{c} \eta \nabla^{c} \eta+\nabla_{c} \omega \nabla^{c} \omega\right),
$$

is divergence free, i.e. $\nabla^{a} T_{a b}=0$.

Eqs. (77)-(9) are purely geometric equations with respect to the metric $h_{a b}$. The essential point is that there exist no dynamical degrees of freedom in 3-dimensional gravity (the Weyl tensor vanishes) and hence all the dynamics is produced by the effective matter sources determined by $(\eta, \omega)$. In other words, the dynamics of vacuum axially symmetric gravitational waves has the behavior of matter moving in a lower dimensional space. Hence, it can be expected that the total mass of the waves can be computed as an "integral on the matter sources". As we will see in the following, this is precisely what happens and it leads to the mass formula in axial symmetry.

\section{$2.12+1$ decomposition}

In order to formulate an initial value problem, we will perform an standard $2+1$ decomposition of Eqs. (7)-(9). Note that this is completely analogous to the $3+1$ decomposition of Einstein equations, in fact all the formulas are formally identical because the dimension does not appear explicitly in them (see, for example, [28], [27]).

Consider a foliation of spacelike, 2-dimensional slices $S$ of the metric $h_{a b}$. Let $t$ be an associated time function and let $n^{a}$ be the unit normal vector orthogonal to $S$ with respect to the metric $h_{a b}$. The intrinsic metric on $S$ is denoted by $q_{a b}$ and is given by

$$
h_{a b}=-n_{a} n_{b}+q_{a b} .
$$


Define the density $\mu$ by

$$
\mu=2^{(3)} R_{a b} n^{a} n^{b}+{ }^{(3)} R
$$

and the current $J_{b}$ by

$$
J_{b}=-q_{b}^{c} n^{a(3)} R_{c a},
$$

where ${ }^{(3)} R={ }^{(3)} R_{a b} h^{a b}$ denotes the trace of ${ }^{(3)} R_{a b}$. Then, using Eq. (9) we obtain

$$
\begin{aligned}
\mu & =\frac{1}{2 \eta^{2}}\left(\eta^{\prime 2}+\omega^{\prime 2}+|D \eta|^{2}+|D \omega|^{2}\right), \\
J_{A} & =-\frac{1}{2 \eta^{2}}\left(\eta^{\prime} D_{A} \eta+\omega^{\prime} D_{A} \omega\right),
\end{aligned}
$$

where $D_{A}$ is the connexion with respect to $q_{A B}$. The prime denotes directional derivative with respect to $n^{a}$, that is

$$
\eta^{\prime}=n^{a} \nabla_{a} \eta=\frac{1}{\alpha}\left(\partial_{t} \eta-\beta^{A} D_{A} \eta\right)
$$

where $\alpha$ is the lapse and $\beta^{A}$ is the shift vector of the foliation. The indices $A, B, \cdots$ denote two dimensional indices on $S$. The constraints equations corresponding to (9) are given by

$$
\begin{aligned}
{ }^{(2)} R-\chi^{A B} \chi_{A B}+\chi^{2} & =\mu \\
D^{A} \chi_{A B}-D_{B} \chi & =J_{B}
\end{aligned}
$$

where ${ }^{(2)} R$ is the Ricci scalar of $q_{A B}, \chi_{A B}$ is the second fundamental form of $S$ and $\chi$ its trace

$$
\chi=q^{A B} \chi_{A B} .
$$

We use the following sign convention for the definition of $\chi_{A B}$

$$
\chi_{a b}=-q_{a}^{c} \nabla_{c} n_{b}=-\frac{1}{2} £_{n} q_{a b},
$$

where $£$ denotes Lie derivative. The evolution equations are given by

$$
\begin{aligned}
\partial_{t} q_{A B} & =-2 \alpha \chi_{A B}+£_{\beta} q_{A B}, \\
\partial_{t} \chi_{A B} & =£_{\beta} \chi_{A B}-D_{A} D_{B} \alpha+\alpha \tau_{A B},
\end{aligned}
$$

where

$$
\tau_{A B}=\chi \chi_{A B}+{ }^{(2)} R_{A B}-{ }^{(3)} R_{A B}-2 \chi_{A C} \chi_{B}^{C}
$$


and

$$
{ }^{(3)} R_{A B}=\frac{1}{2 \eta^{2}}\left(\partial_{A} \eta \partial_{B} \eta+\partial_{A} \omega \partial_{B} \omega\right) .
$$

The evolution equations (21)-(22) and the constraint equations (17)-(18) constitute a complete $2+1$ decomposition of the 3-dimensional Einstein Eq. (9). It remains to decompose the effective matter Eqs. (7)-(8). The result is the following

$$
\begin{aligned}
& -\Sigma^{\prime \prime}+\Delta_{q} \Sigma+D_{A} \Sigma \frac{D^{A} \alpha}{\alpha}+\Sigma^{\prime} \chi=\frac{1}{\eta^{2}}\left(\omega^{\prime 2}-|D \omega|^{2}\right), \\
& -\omega^{\prime \prime}+\Delta_{q} \omega+D_{A} \omega \frac{D^{A} \alpha}{\alpha}+\omega^{\prime} \chi=\frac{2}{\eta^{2}}\left(D_{A} \omega D^{A} \eta-\omega^{\prime} \eta^{\prime}\right),
\end{aligned}
$$

where $\Delta_{q}$ is the Laplacian with respect to $q_{A B}$, i.e. $\Delta_{q}=D^{A} D_{A}$ and we have defined $\Sigma=\log \eta$.

The evolution equations (21) $-(22)$ and $(25)-(26)$ together with the constraint equations (17)-(18) represent the complete $2+1$ decomposition of the geometrical equations (7)-(9) in an arbitrary gauge. This set of equations presents an important feature which is peculiar of $2+1$ dimensions. Namely, the evolution equations (21) -(22) and the constraint equations (17)-(18) are, roughly speaking, equivalent in the following sense. With an appropriate gauge choice (in the next section we will present an important example) the constraint equations (17)-(18) form an elliptic system that determines $q_{A B}$ an $\chi_{A B}$ in terms of the effective matter sources $\eta$ and $\omega$. Hence, the evolution equations (25) -(26) together with the constraint equations (17)-(18) constitute a complete system of equations. In particular, a solution of this system will automatically satisfy the other evolution equations (21)-(22). Alternative, it is possible to solve the evolution equations (25)-(26) and (21) $-(22)$. If the initial data satisfy the constraint equations at a given time, then the solution will satisfy the constraint equations for all times. Note that even when the gauge is fixed, we have different possibilities for constructing evolutions schemes. The first choice is called in the numerical literature "constrained evolution" and the second choice "free evolution". It is also possible to mix them to obtain "mixed evolution" schemes (see the discussion in [29]).

\subsection{Gauge and mass formula}

In this section we describe the maximal-isothermal gauge. In particular we review the mass formula valid in this gauge (see [15] for details). For the lapse, we impose the maximal condition on the 2-surfaces

$$
\chi=0
$$


Note that we are not imposing that the surfaces are maximal in the 3dimensional picture as in [15]. The later condition is the one generally used (see, for example, 6] [30]), but the difference is only minor. In particular the mass formula is positive definite for both conditions. The one used here appears to be natural with respect to the rescaled metric $h_{a b}$. Eq. (27) implies the following well known equation for the lapse

$$
\Delta_{q} \alpha=\alpha\left(\chi^{A B} \chi_{A B}+\mu_{1}\right)
$$

where

$$
\mu_{1}={ }^{(3)} R_{a b} n^{a} n^{b}=\frac{1}{2 \eta^{2}}\left(\eta^{\prime 2}+\omega^{\prime 2}\right) .
$$

The maximal gauge (27) can be, of course, imposed in any dimensions and it is not related at all with axial symmetry. In contrast, the condition for the shift is peculiar for two space dimensions. The shift vector is fixed by the requirement that the intrinsic metric $q_{A B}$ has the following form

$$
q_{A B}=e^{2 u} \delta_{A B}
$$

where $\delta_{A B}$ is a fixed (i.e. $\partial_{t} \delta_{A B}=0$ ) flat metric in two dimensions. Then, using (27), we obtain that the trace free part of (21) is given by

$$
2 \alpha \chi_{A B}=\left(\mathcal{L}_{q} \beta\right)_{A B},
$$

where $\mathcal{L}_{q}$ is the conformal Killing operator in two dimensions with respect to the metric $q_{A B}$. Equation (31) is an elliptic first order system of equations for $\beta^{A}$.

The elliptic Eqs. (28) and (31) determine lapse and shift for the metric $h_{a b}$ and hence they fix completely the gauge freedom in Eqs. (7)-(9). This gauge has associated a natural cylindrical coordinate system $(t, \rho, z)$ for which the metric $\delta_{A B}$ is given

$$
\delta=d \rho^{2}+d z^{2}
$$

and the axis of symmetry is given by $\rho=0$. The slices $S$ are the half planes $\mathbb{R}_{+}^{2}$.

It is useful to introduce the following auxiliary functions. Instead of $\eta$ we will use the function $\sigma$ defined by

$$
\eta=\rho^{2} e^{\sigma}
$$

Note that for Minkowski $\eta=\rho^{2}$, that is, $\sigma$ measure the non-flat part of the norm $\eta$. Also, instead of $u$ it is convenient to work with the function $q$ defined by

$$
u=\log \rho+\sigma+q .
$$


The important property of $q$ is that it vanished at the axis. This is a consequence of the regularity conditions of the metric at the axis (see [20] for details).

The equations presented in section 2.1 can be explicitly written as partial differential equations in the coordinates $(t, \rho, z)$ (see [20]). In particular the Hamiltonian constraint is given by

$$
\text { (3) } \Delta \sigma+\Delta q=-\frac{\epsilon}{4}
$$

where

$$
\epsilon=\frac{e^{2 u}}{\eta^{2}}\left(\eta^{\prime 2}+\omega^{\prime 2}\right)+|\partial \sigma|^{2}+\frac{|\partial \omega|^{2}}{\eta^{2}}+2 e^{-2 u} \hat{\chi}^{A B} \chi_{A B} .
$$

The hat on $\chi^{A B}$ means that the indices are moved with the flat metric $\delta_{A B}$, $\partial$ denotes partial derivatives with respect to $\rho$ and $z, \Delta$ is the 2-dimensional flat Laplacian, namely

$$
\Delta=\partial_{\rho}^{2}+\partial_{z}^{2},
$$

and ${ }^{(3)} \Delta$ is the 3 -dimensional flat Laplacian acting on axially symmetric functions, that is

$$
{ }^{(3)} \Delta=\Delta+\frac{\partial_{\rho}}{\rho} \text {. }
$$

The positive scalar $\epsilon$ plays the role of the energy density of the gravitational waves in this gauge. The integral of $\epsilon$ over the slice $S$ is given by

$$
m=\frac{1}{16} \int_{\mathbb{R}_{+}^{2}} \epsilon \rho d \rho d z .
$$

It is a non-trivial fact that this number $m$ is precisely the total ADM mass of the spacetime. Moreover, the fall off properties of the solutions $\alpha$ and $\beta$ of gauge conditions ensure that the mass is conserved along the evolution, namely

$$
\frac{d}{d t} m=0
$$

see [15] for details.

The mass formula (39) together with the conservation law (40) represent a relevant property of this gauge which is not present in other ones.

In any physical theory conserved quantities (in particular, conserved energies) are very important to control the evolution of the system. However, in General Relativity, the conserved mass appears as a boundary integral and not as a volume integral (as, for example, in the wave equation). Hence it is not possible to relate the mass with any norm of the fields to control the evolution of them (for the wave equation the energy is precisely the norm of the wave). The mass formula (39) for axially symmetric systems in the maximal-isothermal gauge represents a remarkable exception. 


\section{Results}

\subsection{Numerical results}

Axially symmetric spacetimes has been studied numerically since the very beginnings of numerical relativity, see chapter 10.4 in [2] and references therein for an historical perspective. A very good reference for early results is the review article [3]. In particular, in this article the maximal and isothermal gauges are discussed. Another important review article is [26], where the symmetry reductions and $2+1$ decomposition mentioned in section 2 was used for first time in numerical relativity.

As we mentioned in the introduction, the difficulty introduced by the singular behavior at the axis is severe and that made axially symmetric evolution particularly difficult to handle. As a consequence of this "axisymmetric codes were practically abandoned as soon as computers became powerful enough to be able to handle full three-dimensional simulations in the early 1990s." (the quote is from [2]).

New insights in the study of the axial regularity problem in numerical relativity were presented in [23]. They introduced two main ingredients. The first one is that the behavior at the axis is treated as a singular boundary condition which are handled by introducing "ghost zones" outside the axis. The second one is that all the variables used are such that either they vanish at the axis or have vanishing derivative there, but not both. This cure the axis instabilities from the numerical point of view.

Later in [6] a new code was developed which also use similar techniques to handle the axis problem. This article is particularly relevant in our context because they use essentially the same equations and the same gauge conditions as presented in section 2. However, the mass formula (39) for this gauge was not known at that time and hence it was not used in that article.

Further studied were performed in [29] [31], where new evolutions schemes are proposed and the general case with twist is considered. In 32] the regularization procedure at the axis was performed for more general evolutions schemes. In [30] important issues concerning the maximal-isothermal gauge are studied, in particular a new choice of variable is proposed in order to guarantee the uniqueness of solutions of the relevant equations. I will come back to this in the next section.

In a very recent series of articles, the critical collapse of gravitational waves is studied [33] [34]. This code use a different gauge choice, namely harmonic coordinates. Finally, we mention [20] where the linear equations in the maximal-isothermal gauge were studied and boundary conditions compatible with the mass formula were presented. 


\subsection{Analytical results}

The first analytical application of the mass formula is the proof of the following geometrical inequality

$$
\sqrt{|J|} \leq m
$$

where $J$ is the total angular momentum of the spacetime (see [14] [17] for details). Extension of this theorem, which remove technical assumptions and also simplify considerable its proof were presented in [8] [9]. The remarkable aspect of this proof if that extreme Kerr black hole appears as the minimizer of the mass integral. This suggests stability properties of these black holes. In particular, it suggests that it is perhaps easier to prove stability near an extreme black holes than a non-extreme one in axial symmetry (see the essay [16]). There exist important extensions to inequality (41) which include charges [11] [10].

The second geometrical inequality proved with a suitable quasi-local formulation of the mass formula is the following

$$
8 \pi|J| \leq A,
$$

where $A$ is the area and $J$ is the angular momentum of the black hole horizon. See [18], 11] [21] for details. In the stationary case with matter and charge, it has been proved in [24]. Numerical evidences for the validity of this inequality has been presented in [25].

Inequality (42) ensures that the Christodoulou quasi local-mass of the black hole is a monotonically increasing quantity in the evolution (see the discussion in [18]).

Both geometrical inequalities are proven on the initial data, that is, the proofs do not make use of the evolution equations. In this gauge the equations reduce to a coupled hyperbolic-elliptic system which is formally singular at the axis. Due to the rather peculiar properties of the system, the local in time existence has proved to resist analysis by standard methods. To analyze the principal part of the equations, which may represent the main source of the difficulties, we study linear perturbation around the flat Minkowski solution in this gauge. In 22] we solved this linearized system explicitly in terms of integral transformations in a remarkable simple form. This representation is well suited to obtain useful estimates to apply in the non-linear case.

\section{Open Problems}

There exists two relevant open model problems for axially symmetric spacetimes: 
1. Black hole formation and critical collapse of axially symmetric gravitational waves (Numerical).

2. The stability of the Kerr black hole in axial symmetry (Mathematical).

These problems are expected to be difficult, they constitute the long term motivation of this study. In the following I will briefly discuss them in order to propose simpler open problems that can contribute to the resolution of 1 and 2 and at the same time are feasible to solve in the near future.

Most of the numerical work mentioned in section 3.1 is concerned with the vacuum equations and with the critical collapse of the gravitational waves. As we pointed out in the introduction, there exists other interesting physical systems in axial symmetry. For example, the collapse of an axially symmetric rotating star studied in [26]. However, if we restrict ourselves to the vacuum equations (as we do in this article) then probably the most relevant open problem to study in numerical axial symmetry is the critical collapse. And the reason is that this problem can not be handled, with present computers, without symmetry assumptions due to the high resolution needed. The subject has already a long history (see the review article [5]), and despite many efforts the problem still lacks definitive answer (see the recent article [34]).

Although there exist many possible gauge choices in axial symmetry, the mass formula suggests the maximal-isothermal gauge as privileged one. Also, constrained evolutions schemes appear to be better than free evolutions schemes (see the discussion in [30]). In the following we focus on the maximal-isothermal gauge in its constrained formulation. In the article [30] it was clearly pointed out one of the main problems of these equations. There are many elliptic equations and it is not clear a priori that they will always admit a unique solution along the evolution. A new set of variables was proposed in that article to ensure that the equations have the correct behavior at the linearized level to ensure uniqueness of solution. However, it is still not clear if the non-linear equations (notably, the Hamiltonian constraint) is always solvable. It is an open problem to see if these equations (or some variant of them) are well posed. I believe that this is an important problem that stress the interplay between analytical and numerical studies. Perhaps the resolution of the well-posedness question will lead us to select (or even discover) the correct evolution scheme.

Another question which is important in numerical evolution is the issue of outer boundary conditions on a finite domain. In the article [20] a set of boundary condition at the linear level were proposed. This boundary conditions have the property that the energy appears to leave the domain, at least for the class of initial data studied in this article. It is important to note that 
the mass formula provides a well-defined local measure of the gravitational energy. These boundary conditions have not yet been implemented for the complete equations.

The following two problems summarize the discussion above.

1.a. Well-posedness for the maximal isothermal gauge. Is the Hamiltonian constraint always solvable? Does the gauge breaks down in the strong field regime?

\section{1.b. Implementation of the radiation boundary conditions for the} full equations. How much they improve the evolution scheme? It is possible to prove (may be with some extra assumptions) that for such boundary conditions the energy always leaves the domain?

As we have seen, problem 1 is more numerically oriented, since it appears to be very difficult to understand critical phenomena with the current analytical tools. In contrast problem 2 is more analytical. In recent years there have been important new developments in the problem of the linear stability of the Kerr black hole from the analytical point of view. Most of this work is concerned with scalar waves on a fixed Kerr black hole background. We refer to the review articles [12] and [13] and reference therein. These works are not restricted to axial symmetry. The expectation is, of course, that axially symmetric spacetimes are simpler. In particular, axial symmetry provides extra geometrical structure that can give new insight into the equations. As we mentioned in section 3.2 much of the current evidences that this is the case come from the geometrical inequalities (41) and (42). These inequalities have been proved under some restricted conditions. In the case of the quasi-local inequality (42), the only restriction is that the initial data are assumed to be maximal. It is important to remove this restriction or to find counter examples. Numerics can play a relevant role in the search of possible counter examples. Also, it would be very interesting to generalize this inequality to include the charge. This is important not only because the charge is a relevant parameter of a black hole but also because the charge is well defined without any symmetry assumption (this is not the case of the quasi-local angular momentum). The natural question is whether a similar inequality holds without axial symmetry. This would involve an appropriate definition of quasi-local angular momentum. Another interesting problem is to study what happens in the case of equality in (42). A rigidity property has been proved in [21] that characterize the local geometry of the surface in that case. However, it is very likely that this kind of surface can only appear as asymptotic limit as the extreme Kerr initial data. A related question 
is to study small deformation of the extreme Kerr initial data, as the ones constructed in [19]. Do these initial conditions admit apparent horizons if the perturbation is small enough? This question is relevant because if the answer is negative then these initial conditions have the chance to provide complete Cauchy surfaces that are outside the black hole region, as in the extreme Kerr black hole.

Regarding the global inequality (41), the most relevant open problems is to prove it for multiple black holes and also to remove the maximal condition. The multiple black holes case is related with the uniqueness of the Kerr black hole among stationary black holes with disconnected horizon components.

All the previous problems are concerned with the initial conditions. The maximal-isothermal gauge will be useful to answer questions regarding the black hole stability if the mass formula can be used in some way to control the evolution. A natural first problem would be to recover the non-linear stability of Minkowski [7] in this gauge. The expectation is that the mass formula will provide a simpler (and different) kind of approach to this problem; although, of course, always restricted to axial symmetry. A second problem it is the study of axially symmetric perturbations of a black hole in this gauge. The expectation is that the mass formula can help to prove linear stability under axially symmetric perturbations of the Kerr black hole. The advantage of this problem in comparison with the non-linear stability of Minkowski in this gauge is that it is probably easier to deal with and also it will prove something new, since the linear stability of the Kerr black hole is still an open question.

We summarize this discussion in the following list of open problems in relation to problem 2 .

2.a. Geometrical inequalities. Remove the maximal assumption. For the quasi-local inequality (42) include the charge and study the possible generalization of this inequality without any symmetry assumptions. Study the case of the equality: it is possible to have such a surface in an asymptotically flat initial data? Study the existence of minimal surfaces or apparent horizon for small perturbation of extreme Kerr initial data. For the global inequality (41), prove it for multiples black holes.

2.b. Linear stability of the Kerr black hole in axial symmetry. Use the mass conservation to control the linear evolution. 


\section{References}

[1] Andrés Aceña, Sergio Dain, and María E Gabach Clément. Horizon areaangular momentum inequality for a class of axially symmetric black holes. Classical and Quantum Gravity, 28(10):105014, 2011, 1012.2413.

[2] Miguel Alcubierre. Introduction to 3+1 numerical relativity, volume 140 of International Series of Monographs on Physics. Oxford University Press, Oxford, 2008.

[3] J. M. Bardeen and T. Piran. General relativistic axisymmetric rotating systems: coordinates and equations. Physics Reports, 96:205-250, 1983.

[4] J. Bičák and A. Pravdová. Symmetries of asymptotically flat electrovacuum space-times and radiation. Journal of Mathematical Physics, 39(11):6011-6039, 1998.

[5] José M. Martín-García Carsten Gundlach. Critical phenomena in gravitational collapse. Living Reviews in Relativity, 10(5), 2007.

[6] Matthew W. Choptuik, Eric W. Hirschmann, Steven L. Liebling, and Frans Pretorius. An Axisymmetric Gravitational Collapse Code. Class. Quant. Grav., 20:1857-1878, 2003, gr-qc/0301006.

[7] D. Christodoulou and S. Klainerman. The Global Nonlinear Stability of the Minkowski Space, volume 41 of Princeton Mathematical series. Princeton University Press, Princeton, NJ, 1993.

[8] Piotr T. Chrusciel. Mass and angular-momentum inequalities for axisymmetric initial data sets I. Positivity of mass. Annals Phys., 323:25662590, 2008, 0710.3680.

[9] Piotr T. Chruściel, Yanyan Li, and Gilbert Weinstein. Mass and angularmomentum inequalities for axi-symmetric initial data sets. II. Angularmomentum. Ann. Phys., 323(10):2591-2613, 2008, arXiv:0712.4064.

[10] Piotr T. Chrusciel and Joao Lopes Costa. Mass, angular-momentum, and charge inequalities for axisymmetric initial data. Class. Quant. Grav., 26:235013, 2009, 0909.5625.

[11] Joao Lopes Costa. Proof of a Dain inequality with charge. Journal of Physics A: Mathematical and Theoretical, 43(28):285202, 2010, 0912.0838. 
[12] Mihalis Dafermos and Igor Rodnianski. Lectures on black holes and linear waves, 2008, 0811.0354.

[13] Mihalis Dafermos and Igor Rodnianski. The black hole stability problem for linear scalar perturbations. 2010, 1010.5137.

[14] Sergio Dain. Angular momemtum-mass inequality for axisymmetric black holes. Phys. Rev. Lett., 96:101101, 2006, gr-qc/0511101.

[15] Sergio Dain. Axisymmetric evolution of Einstein equations and mass conservation. Class. Quantum. Grav., 25:145021, 2008, 0804.2679.

[16] Sergio Dain. The inequality between mass and angular momentum for axially symmetric black holes. International Journal of Modern Physics D, 17(3-4):519-523, 2008, arXiv:0707.3118 [gr-qc].

[17] Sergio Dain. Proof of the angular momentum-mass inequality for axisymmetric black holes. J. Differential Geometry, 79(1):33-67, 2008, gr-qc/0606105.

[18] Sergio Dain. Extreme throat initial data set and horizon area-angular momentum inequality for axisymmetric black holes. Phys. Rev. D, 82(10):104010, Nov 2010, 1008.0019.

[19] Sergio Dain and Maria E. Gabach Clément. Small deformations of extreme Kerr black hole initial data. Class. Quant. Grav., 28:075003, 2011, 1001.0178 .

[20] Sergio Dain and Omar E. Ortiz. Well-posedness, linear perturbations, and mass conservation for the axisymmetric einstein equations. Phys. Rev. D, 81(4):044040, Feb 2010.

[21] Sergio Dain and Martin Reiris. Area - Angular momentum inequality for axisymmetric black holes, 2011, 1102.5215.

[22] Sergio Dain and Martin Reiris. Linear perturbations for the vacuum axisymmetric Einstein equations. Annales Henri Poincare, 12:49-65, $2011,1005.5347$.

[23] David Garfinkle and G. Comer Duncan. Numerical evolution of Brill waves. Phys. Rev., D63:044011, 2001, gr-qc/0006073.

[24] Jörg Hennig, Carla Cederbaum, and Marcus Ansorg. A universal inequality for axisymmetric and stationary black holes with surrounding matter in the Einstein-Maxwell theory. Commun. Math. Phys., 293:449467, 2010, 0812.2811. 
[25] Jose Luis Jaramillo, Nicolas Vasset, and Marcus Ansorg. A numerical study of Penrose-like inequalities in a family of axially symmetric initial data. 2007, 0712.1741.

[26] T. Nakamura, K. Oohara, and Y. Kojima. General Relativistic Collapse to Black Holes and Gravitational Waves from Black Holes. Progress of Theoretical Physics Supplement, 90:1-218, 1987.

[27] Alan Rendall. 3+1. http://www.aei.mpg.de/ rendall/3+1.ps, 2008.

[28] Alan D. Rendall. Partial differential equations in general relativity, volume 16 of Oxford Graduate Texts in Mathematics. Oxford University Press, Oxford, 2008.

[29] Oliver Rinne. Axisymmetric Numerical Relativity. PhD thesis, University of Cambridge, 2005, gr-qc/0601064.

[30] Oliver Rinne. Constrained evolution in axisymmetry and the gravitational collapse of prolate Brill waves. Class. Quantum. Grav., 25:135009, 2008, 0802.3791.

[31] Oliver Rinne and John M. Stewart. A strongly hyperbolic and regular reduction of Einstein's equations for axisymmetric spacetimes. Class. Quantum. Grav., 22:1143-1166, 2005, gr-qc/0502037.

[32] Milton Ruiz, Miguel Alcubierre, and Dario Nunez. Regularization of spherical and axisymmetric evolution codes in numerical relativity. Gen. Rel. Grav., 40:159-182, 2008, 0706.0923.

[33] Evgeny Sorkin. An Axisymmetric generalized harmonic evolution code. Phys.Rev., D81:084062, 2010, 0911.2011.

[34] Evgeny Sorkin. On critical collapse of gravitational waves. Class.Quant.Grav., 28:025011, 2011, 1008.3319. 